\title{
Beneficial effects of weight loss associated with moderate calorie/carbohydrate restriction, and increased proportional intake of protein and unsaturated fat on serum urate and lipoprotein levels in gout: a pilot study
}

\author{
P H Dessein, E A Shipton, A E Stanwix, B I Joffe, J Ramokgadi
}

Rheumatology Unit, Milpark Hospital, Pain Relief and Research

Unit, CH Baragwanath Hospital, University of Witwatersrand,

Johannesburg, South

Africa

P H Dessein

Pain Relief and

Research Unit, $\mathbf{C H}$

Baragwanath Hospital

E A Shipton

Department of

Rheumatology,

Johannesburg

Hospital, University of

Witwatersrand,

Johannesburg, South

Africa

A E Stanwix

Carbohydrate and Lipid Metabolism Research Unit, Johannesburg Hospital B I Joffe

Department of

Quantitative

Management, UNISA,

Pretoria, South Africa

J Ramokgadi

Correspondence to:

Dr P Dessein, PO Box 1012 ,

Melville 2109, Johannesburg,

South Africa

Email: Dessein@elink.co.za

Accepted for publication

20 January 2000

\begin{abstract}
Objectives-Insulin resistance (IR) has been increasingly implicated in the pathogenesis of gout. The lipoprotein abnormalities described in hyperuricaemic subjects are similar to those associated with IR, and insulin influences renal urate excretion. In this study it was investigated whether dietary measures, reported to be beneficial in IR, have serum uric acid (SU) and lipid lowering effects in gout. Methods-Thirteen non-diabetic men (median age 50, range 38-62) were enrolled. Each patient had had at least two gouty attacks during the four months before enrolment. Dietary recommendations consisted of calorie restriction to $6690 \mathrm{~kJ}(1600 \mathrm{kcal})$ a day with $40 \%$ derived from carbohydrate, $30 \%$ from protein, and $30 \%$ from fat; replacement of refined carbohydrates with complex ones and saturated fats with mono- and polyunsaturated ones. At onset and after 16 weeks, fasting blood samples were taken for determination of SU, serum cholesterol (C), low density lipoprotein cholesterol (LDL-C), high density lipoprotein cholesterol (HDL-C), and triglycerides (TGs). Results were expressed as median (SD).
\end{abstract}

Results-At onset, the body mass index (BMI) was $30.5(8.1) \mathrm{kg} / \mathrm{m}^{2}$. Dietary measures resulted in weight loss of $7.7(5.4) \mathrm{kg}$ $(p=0.002)$ and a decrease in the frequency of monthly attacks from $2.1(0.8)$ to 0.6 $(0.7)(p=0.002)$. The SU decreased from $0.57(0.10)$ to $0.47(0.09) \mathrm{mmol} / 1(p=0.001)$ and normalised in $7(58 \%)$ of the 12 patients with an initially raised level. Serum cholesterol decreased from $\mathbf{6 . 0}$ (1.7) to $4.7(0.9) \mathrm{mmol} / 1(\mathrm{p}=0.002), \mathrm{LDL}-\mathrm{C}$ from $3.5(1.2)$ to $2.7 \quad(0.8) \quad \mathbf{m m o l} / 1$ $(p=0.004)$, TGs from $4.7(4.2)$ to $1.9(1.0)$ mmol/1 $(p=0.001)$, and C:HDL-C ratios from $6.7(1.7)$ to $5.2(1.0) \quad(p=0.002)$. HDL-C levels increased insignificantly. High baseline SU, frequency of attacks, total cholesterol, LDL-C and TG levels, and total C:HDL-C ratios correlated with higher decreases in the respective variables upon dietary intervention $(p<0.05)$. Conclusion-The results suggest that weight reduction associated with a change in proportional macronutrient intake, as recently recommended in IR, is beneficial, reducing the SU levels and dyslipidaemia in gout. Current dietary recommendations for gout may need re-evaluation. (Ann Rheum Dis 2000;59:539-543)

Gout and the insulin resistance (IR) syndrome have several features in common. ${ }^{1}$ Insulin resistance is associated with obesity, hypertension, glucose intolerance, coronary artery disease (CAD), increased triglyceride (TG) levels, decreased high density lipoprotein cholesterol (HDL-C) concentrations, increased C:HDL-C ratios, low density lipoprotein cholesterol (LDL-C), and uric acid levels, and atherogenic small, dense LDL-C particles. ${ }^{2}{ }^{3}$ Hyperuricaemia is a likely feature of the IR syndrome. ${ }^{2}{ }^{4}$ Indeed, high serum uric acid (SU) levels are associated with high fasting insulin, serum TGs, LDL-C, diastolic blood pressure, and C:HDL-C ratios. ${ }^{5}$

Current dietary recommendations for gout entail limitation of purine, protein, and alcohol intake, and weight reduction. ${ }^{6-8}$ Foods containing a low purine content are often high in carbohydrate and saturated fat. Weight reduction enhances insulin sensitivity and lowers insulin and TG levels. ${ }^{9}$ In addition, the proportional intake of macronutrients affects insulin sensitivity. A low energy, high protein diet improves insulin sensitivity, whereas a low energy, high carbohydrate diet decreases insulin sensitivity. ${ }^{10}$ Although substitution of saturated fats by carbohydrate leads to a decrease in total cholesterol and LDL-C in subjects with IR, this strategy has been associated with increased insulin, glucose, TG, and small, dense LDL-C particle concentrations, and decreased HDL-C levels. ${ }^{9}$ On the other hand, unsaturated dietary fats similarly reduce total cholesterol and LDL-C, ${ }^{11}$ and a high monounsaturated/low carbohydrate diet enhances insulin sensitivity with decreases in postprandial glucose and plasma insulin, and fasting plasma TG levels. ${ }^{12}$ Also, increased intake of n-3 fatty acids is associated with a reduction in mortality in patients with CAD. ${ }^{13}$ Atherosclerosis is common in gout and this has been attributed to clinical correlates, such as obesity, dyslipidaemia, hypertension, and diabetes mellitus. ${ }^{4}$

In view of the above, IR may be a modifiable pathogenetic mechanism in gout and its metabolic complications. ${ }^{78}$ In this study we 
Table 1 Baseline characteristics in 13 patients with gout

\begin{tabular}{ll}
\hline Characteristic & \\
\hline Median (SD; range) age in years & $50(5.6 ; 38-62)$ \\
Median (SD; range) disease duration in & $7(10.2 ; 0.5-38)$ \\
$\quad$ years & $10(77)$ \\
Number (\%) of alcohol users & $7(54)$ \\
Number (\%) of hypertensive patients & \\
\hline
\end{tabular}

investigated whether weight loss through moderate limitation of calorie and carbohydrate intake, together with an increased proportional intake of protein and replacement of saturated by unsaturated fat, is of therapeutic benefit in this condition.

\section{Methods}

Thirteen white men with acute intermittent gout, confirmed by compensated polarised microscopy, were enrolled. Exclusion criteria were biochemical evidence of diabetes, hypothyroidism or renal impairment, CAD, alcohol consumption in excess of $25 \mathrm{~g}$ a day, and current use of equal or less carbohydrate or saturated fat than recommended in the diet. Patients who had had fewer than two gout attacks over the previous four months or felt they could not accurately remember the number of gout attacks they had had in the previous four months were also excluded. None of the patients had tophi or a history of nephrolithiasis. Investigations to identify intrinsic overproduction or underexcretion of uric acid were not performed. ${ }^{6}$ Acute attacks were treated with diclofenac sodium, and only three patients were taking other drugs: enalapril (one patient), verapamil (one patient), and atenolol and lisinopril (one patient). None was taking hypouricaemic agents. No patient participated in sports. The number of attacks over the previous four months was recorded and the body mass index (BMI) $\left(\mathrm{kg} / \mathrm{m}^{2}\right)$ was calculated for each patient. At enrolment, fasting blood samples were taken (between 0800 and 1000) for determination (using autoanalysers and enzymatic methods (for lipids)) of SU; total cholesterol, HDL-C, and TGs (Olympus Diagnostics, Ireland); and LDL-C (Randox Laboratories Ltd, United Kingdom). In one patient LDL-C and HDL-C could not be measured at enrolment owing to interference by a markedly raised TG level.

Dietary intervention was designed to comprise $6690 \mathrm{~kJ}(1600 \mathrm{kcal}$ ) a day with $40 \%$ energy derived from carbohydrate, $30 \%$ from protein, and $30 \%$ from fat, and each meal containing similar macronutrient proportions to

Table 2 Median (SD) clinical and laboratory features at enrolment and after 16 weeks of dietary intervention, and respective median decreases in 13 patients with gout

\begin{tabular}{lllll}
\hline Clinical/laboratory feature & At enrolment & At 16 weeks & Decrease (range) & p Value \\
\hline Weight $(\mathrm{kg})$ & $91.1(23.5)$ & $83.4(22.0)$ & $7.7(0-21)$ & 0.002 \\
BMI $^{2}\left(\mathrm{~kg} / \mathrm{m}^{2}\right)$ & $30.5(8.1)$ & $27.8(7.9)$ & $2.7(0-6.7)$ & 0.002 \\
Attacks per month & $2.1(0.8)$ & $0.6(0.7)$ & $1.5(0-2.5)$ & 0.002 \\
Uric acid (mmol/l) & $0.57(0.10)$ & $0.47(0.09)$ & $0.10(0.03-0.16)$ & 0.001 \\
Total cholesterol (mmol/l) & $6.0(1.7)$ & $4.7(0.90)$ & $1.3(0.3-5.5)$ & 0.002 \\
LDL-C ${ }^{\star}(\mathrm{mmol} / \mathrm{l})$ & $3.5(1.2)$ & $2.7(0.80)$ & $0.8(-0.01-3.66)$ & 0.004 \\
HDL-C $(\mathrm{mmol} / \mathrm{l})$ & $0.87(0.21)$ & $0.91(0.16)$ & $-0.04(-0.24-0.09)$ & 0.5 \\
C:HDL-C ratio & $6.7(1.7)$ & $5.2(1.0)$ & $1.5(0.3-5.6)$ & 0.002 \\
TG* (mmol/l) & $4.7(4.2)$ & $1.9(1.0)$ & $2.8(0.19-11.7)$ & 0.001 \\
\hline
\end{tabular}

${ }^{\star} \mathrm{BMI}=$ body mass index; LDL-C $=$ low density lipoprotein cholesterol; HDL-C = high density lipoprotein cholesterol; TG $=$ triglyceride. those recommended by Sears. ${ }^{14}$ Patients were advised to have three to five meals a day, replace refined carbohydrates with complex ones, saturated fat with monounsaturated fat (macadamia nuts, almonds, peanuts and peanut butter, olive oil and olive oil margarine, canola oil and canola oil margarine, and avocados), and to have polyunsaturated fat (fish) at least four times a week. The rationale for each of these instructions was explained.

After the initial visit, patients were followed up after 4,10, and 16 weeks subsequent to enrolment. At each visit the number of gout attacks was recorded, the diet was evaluated by 24 hour recall, and further counselling was given. Alcohol intake remained unaltered, and in view of its limited intake in this patient cohort, this was not taken into account when designing dietary instructions. During the last visit, the BMI was calculated and fasting blood samples were taken for determination of SU and lipids.

The data were analysed by the Wilcoxon signed rank test and Pearson correlation coefficients. Results were expressed as median (SD).

\section{Results}

Table 1 presents the baseline features of the patients. Ten $(77 \%)$ of the patients used alcohol, between 12.5 and $21.4 \mathrm{~g}$ a day, and none of them smoked.

Table 2 shows the body weight, BMI, attack frequency, SU levels, and lipid values at enrolment and after 16 weeks' dietary intervention together with the respective changes. At onset, the BMI was normal in one $(8 \%)$ patient, six $(46 \%)$ patients were overweight (BMI $>25$ and $\left.<30 \mathrm{~kg} / \mathrm{m}^{2}\right)$, and six (46\%) were obese. After 16 weeks the BMI was normal in two (15\%) patients, in the overweight range in seven (54\%), while four $(31 \%)$ remained obese. The SU was raised $(>0.51 \mathrm{mmol} / \mathrm{l})$ in $11(85 \%)$ patients at enrolment and subsequently decreased in each patient, with normal values being recorded in eight $(62 \%)$ of them. Weight loss and a decrease in the frequency of gout attacks (fig 1) occurred in all except one patient. The SU and the lipid values of this latter patient did, however, improve (to SU 0.08 $\mathrm{mmol} / \mathrm{l}$ ), and his attacks were shorter and less severe. Each patient had an attack at enrolment. At the final visit only four $(31 \%)$ had an attack. Seven (54\%) of the patients were hypertensive. Of the four who did not take antihypertensive drugs, a normal blood pressure was recorded in two at 16 weeks.

Two patients had a family history of premature CAD. According to the National Cholesterol Education Program (NCEP), ${ }^{15}$ cholesterol or LDL-C, or both, were markedly raised (above the threshold for lipid lowering drug treatment) in seven $(54 \%)$ of the patients at enrolment compared with one $(8 \%)$ patient after dietary intervention. TG levels were initially raised $(>2.3 \mathrm{mmol} / \mathrm{l})$ in nine $(69 \%)$ (range 2.33-15.65 mmol/l) patients compared with three $(23 \%)$ (range $2.56-3.85 \mathrm{mmol} / \mathrm{l}$ ) patients after 16 weeks. At enrolment, HDL-C levels were $<1.2 \mathrm{mmol} / 1$ (median in the male population $)^{16}$ in each patient and $<0.91 \mathrm{mmol} / 1$ 

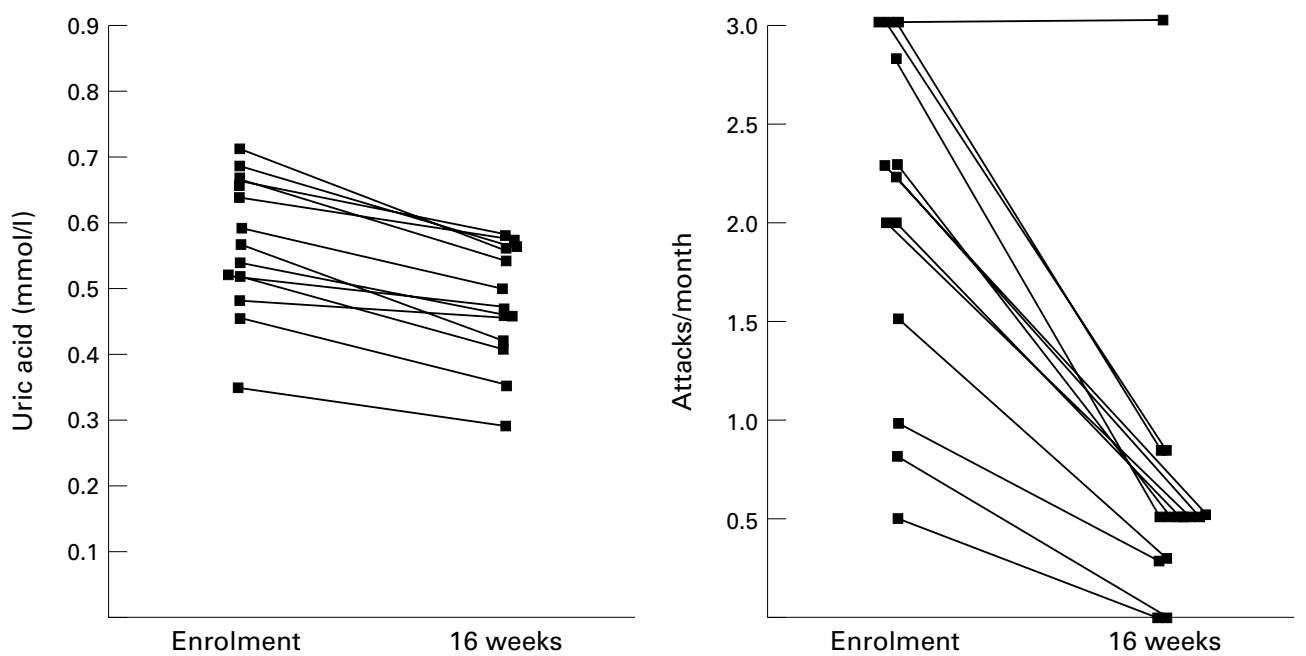

Figure 1 Serum uric acid concentrations and monthly gouty attack frequency at enrolment and 16 weeks.

(associated with increased risk for CAD) ${ }^{15}$ in six $(46 \%)$ patients. HDL-C levels improved insignificantly after dietary intervention. Initial C:HDL-C ratios were above 5 (range 5.310.1) (associated with increased risk for CAD $)^{17}$ in all patients, and six (46\%) patients were at immediate risk for CAD. ${ }^{18}$ In four of the latter, cholesterol and LDL-C were normal. After dietary intervention the C:HDL-C ratio decreased to below 5 in seven $(54 \%)$ patients, and two $(15 \%)$ patients were still at immediate risk for CAD. In one of the latter patients, cholesterol and LDL-C were still raised.

A high SU at enrolment correlated with a large decrease in SU after dietary intervention $(r=0.599, \mathrm{p}=0.032)$ (fig 1$)$. Such correlations were also found for the frequency of attacks $(r=0.584, \quad \mathrm{p}=0.036) \quad($ fig 1$)$, cholesterol $(r=0.832, \quad \mathrm{p}<0.0001), \quad$ LDL-C $\quad(r=0.745$, $\mathrm{p}=0.005)$, C:HDL-C ratios $(r=0.770$, $\mathrm{p}=0.003)$ (fig 2$)$, and TG $(r=0.989, \mathrm{p}<0.0001)$ (fig 2). A similar trend was found between the BMI at enrolment $v$ a decrease in BMI after intervention $(r=0.512, \mathrm{p}=0.074)$.

At enrolment, a high triglyceride level correlated with a high C:HDL-C ratio $(r=0.721$, $p=0.008)$. This relation was no longer present

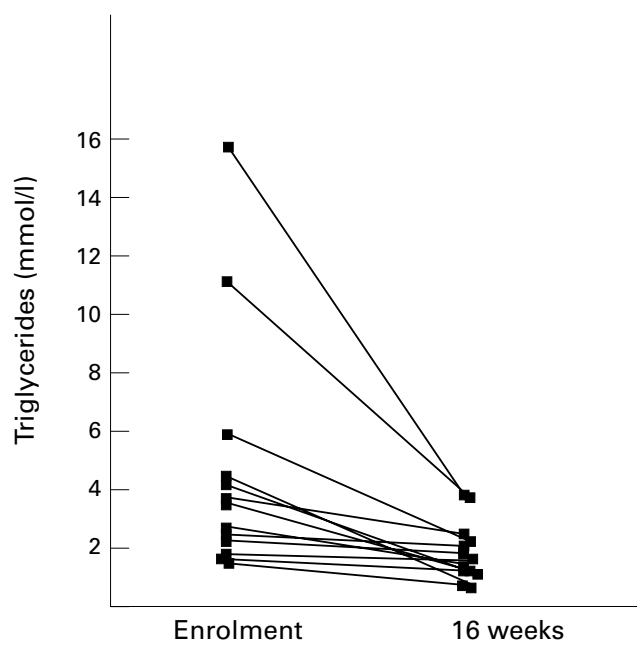

after dietary intervention $(r=0.113, \mathrm{p}=0.714)$. No other significant correlations between the features of the IR syndrome were found, which might be owing to the small number of patients in this study. Also, although a large decrease in BMI was numerically associated with a larger reduction in SU $(r=0.254)$, this was not significant $(\mathrm{p}=0.40)$.

At the time of writing, further follow up data are available for nine patients for a median period of 12 (range 3-14) months; BMI, SU, and lipid values did not change significantly (results not shown). Five patients had no further gout attacks and three had one episode each. One patient, whose SU was $0.35 \mathrm{mmol} / 1$ at enrolment, sustained three attacks over a three months period after he had regained his initial weight. This was accompanied by an increase in SU from 0.29 to $0.34 \mathrm{mmol} / 1$.

\section{Discussion}

In this study we recorded a significant decrease in mean SU levels of $18 \%$ in 13 patients with gout after four months of dietary intervention. This was accompanied by a $67 \%$ reduction in monthly gout attack frequency. The recommended protein intake was $120 \mathrm{~g}$ daily as

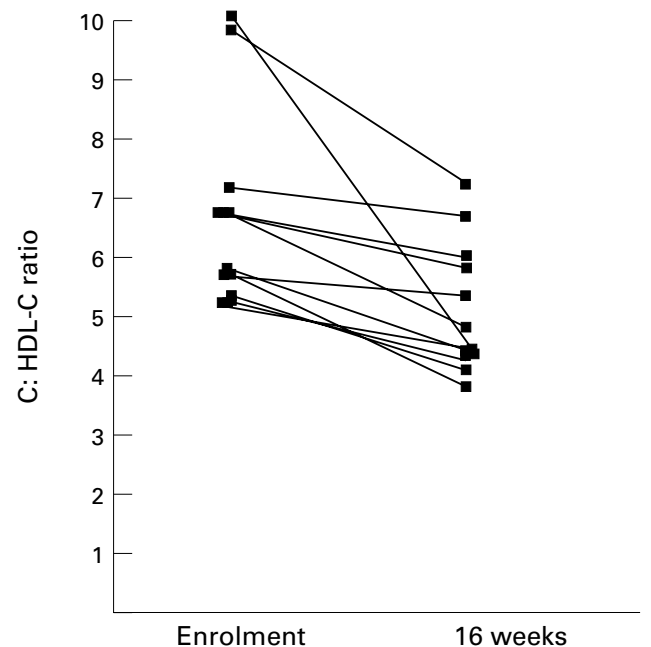

Figure 2 Serum triglyceride concentrations and C:HDL-C ratios at enrolment and after 16 weeks. 
compared with the 90-95 g daily generally used in the Western population. ${ }^{19}$ Moreover, patients were encouraged to limit dairy products (low in purine content), ${ }^{4}$ such as cheese, as a source of protein in view of their high saturated fat content, and increase their intake of poultry and fish, such as salmon, haddock, trout, and mackerel. As the latter are relatively high in purine content, ${ }^{4}$ and no patient decreased their meat intake, dietary measures as used in this study did not limit purine intake.

In a previous study a weight loss of $8 \mathrm{~kg}$ was associated with an $11 \%$ decrease in uric acid levels. ${ }^{20}$ In three of the 15 patients who participated, uric acid concentrations remained constant. ${ }^{20}$ The authors reported a significant correlation between weight loss and fall in uric acid levels. ${ }^{20}$ The latter correlation did not reach significance in our patient cohort, and the SU level decreased in each of our patients. Although this might be owing to the small number of patients enrolled, the macronutrient proportions used in our study would also be expected to contribute to a decrease in SU levels. Thus limitation of carbohydrate intake, ${ }^{12}$ an increased proportional intake of protein, ${ }^{10}$ and the use of unsaturated fat ${ }^{11}$ have each been shown to enhance insulin sensitivity. The one patient who failed to lose weight in this study had a reduction in $\mathrm{SU}$ of $0.08 \mathrm{mmol} / 1$ despite a constant protein and purine intake. This suggests weight loss is not the only factor mediating a therapeutic response.

Insulin stimulates the renal tubular sodiumhydrogen exchanger thereby facilitating secretion of hydrogen and reabsorption of not only sodium, bicarbonate, and chloride but also organic anions such as urate. This constitutes a putative mechanism by which IR and hyperinsulinaemia cause hyperuricaemia. ${ }^{21}$ In keeping with the latter, IR is inversely related to the urinary uric acid clearance and the latter is inversely related to SU concentrations. ${ }^{22}$ Indeed, SU may serve as a surrogate marker of IR. ${ }^{1}$ Although raised TG levels are a hallmark of $\operatorname{IR}^{9}$ and appear to exist intrinsically in gout, ${ }^{23}$ a low calorie diet in hyperuricaemichypertriglyceridaemic patients resulted in a reduction in serum TG concentrations together with an increased uric acid clearance and a decrease in SU levels, which did not occur in normolipidaemic-hyperuricaemic patients. ${ }^{24}$ We recently reported raised insulin levels and IR in $20(95 \%)$ and $16(76 \%)$ of 21 patients with gout, respectively. ${ }^{25}$ In a ongoing study, dietary measures as used in the present patient cohort, resulted in a median decrease in fasting insulin levels and increase in insulin sensitivity (estimated by the homeostasis model assessment) ${ }^{26}$ of $36 \%$ (range 16-63) and $38 \%$ (range 17-64), respectively. Nine overweight/obese patients with a variety of rheumatic disorders (gout in two patients) have been investigated so far (unpublished). Our findings suggest that the dietary measures used in this study are beneficial through an enhancement of insulin sensitivity.

The high incidence of obesity, hypertension, and raised TG levels found in our patients, is in keeping with previous reports. ${ }^{4}$ Recently, lipo- protein abnormalities have been found reliable in predicting CAD risk, ${ }^{15} 18$ and gout is associated with atheroclerosis. ${ }^{4}$ According to the $\mathrm{NCEP}^{15}$ and the Sheffield table for primary prevention of cardiovascular disease, ${ }^{18} 11$ $(85 \%)$ of our patients were at increased risk at enrolment as compared with only three $(23 \%)$ patients after dietary intervention. Also, high TG levels constitute an independent risk factor for $\mathrm{CAD}^{27}$ and when combined with raised C:HDL-C or LDL-C:HDL-C ratios are associated with a fivefold increase in CAD risk. ${ }^{27}{ }^{28}$ The co-occurrence of raised TG and C:HDL-C ratios was present in nine $(69 \%)$ patients at enrolment as compared with in only two $(15 \%)$ patients after dietary intervention. The higher the baseline SU, cholesterol, TG, and C:HDL-C ratios, the higher the respective reductions were upon dietary intervention. Statin treatment has been reported to have a similar effect on hypertriglyceridaemia. ${ }^{16}$

Long term compliance with dietary restriction is the most difficult aspect of the management of obesity and the significant $67 \%$ decrease in the frequency of attacks may not be clinically relevant in view of the short duration of our study. Therefore, the findings that the BMI, SU, and lipid values remained constant in eight of the nine patients during a median follow up period of 12 months, is encouraging. In addition, this was associated with a further decrease in the frequency of attacks.

In conclusion, weight reduction with a change in the proportional intake of macronutrients, as recently recommended for subjects with IR, ${ }^{9}{ }^{10} 12$ is associated with a decrease in SU levels, frequency of attacks, and has beneficial effects on dyslipidaemia in gout. Although a reduction in SU levels suggests that insulin sensitivity is enhanced, further studies-for example, by euglycaemic clamp testing, are needed to confirm this. Current dietary recommendations for gout entail, apart from limitation of alcohol intake and weight loss, limitation of protein, and the unlimited use of several food substances high in carbohydrate or saturated fat. ${ }^{29}$ In view of the high incidence of atherosclerosis and atherogenic lipoprotein patterns, and the beneficial effects seen in our study, we believe that current dietary recommendations for gout may need re-evaluation.

Funding: This study was supported by Lancet Laboratories, Johannesburg, South Africa.

1 Vuorinen-Markkola H, Yki-Jarvinen H. Hyperuricemia and insulin resistance. J Clin Endocrinol Metab 1994;78:25-9. Davidson MB. Clinical implications of insulin resistance syndrome. Am J Med 1995;99:420-6.

3 Jeppesen J, Facchini FS, Reaven GM. Individuals with high total cholesterol/HDL ratios are insulin resistant. J Intern Med 1998;243:293-8.

4 Kelly WN, Wortmann RL. Gout and hyperuricemia. In: Kelly WN, Harris ED, Ruddy S, Sledge CB, eds. Textbook of rheumatology. Philadelphia: Saunders, 1997:1313-51.

5 Cigolini M, Targher G, Tonoli M, Manara F, Muggeo M, De Sandre G. Hyperuricemia: relationships to body fat distribution and other components of the insulin resistance syndrome in 38-year-old healthy men and women. Int J Obes Relat Metab Disord 1995;19:92-6.

6 Emmerson BT. The management of gout. N Engl J Med 1996;334:445-51.

7 Emmerson BT. Hyperlipidaemia in hyperuricaemia and gout. Ann Rheum Dis 1998;57:509-10.

8 Pascual E. Management of crystal arthritis. Rheumatology 1999;38:912-16.

9 Reaven GM. Do high carbohydrate diets prevent the development or attenuate the manifestations (or both) of 
syndrome X? A viewpoint strongly against. Curr Opin Lipidol 1997;8:23-7.

10 Piatti PM, Monti F, Fermo I, Baruffaldi L, Nasser R, Santambrogio G, et al. Hypocaloric high-protein diet improves glucose oxidation and spares comparison to hypocaloric high-carbohydrate diet. Metabolism 1994;43:1481-7

11 Gardner CD, Kraemer HC. Monounsaturated versus polyunsaturated dietary fat and serum lipids. A meta-analysis. Arterioscler Thromb Vasc Biol 1995;15:1917-27.

12 Parillo M, Rivellese AA, Ciardullo AV, Capaldo D, Giacco A, Genovese S, et al. A high-monounsaturated-fat/lowcarbohydrate diet improves peripheral insulin sensitivity in non-insulin-dependent diabetic patients. Metabolism 1992;41:1373-8.

13 de Lorgeril M, Renaud S, Mamelle N, Salen P, Martin J-L, Monjaud I, et al. Mediterranean alpha-linolenic acid-rich diet in secondary prevention of coronary heart disease. diet in secondary preventio

14 Sears B, Lawren B. Enter the zone. New York: Harper Collins, 1995.

15 Expert Panel on Detection, Evaluation, and Treatment of High Blood Cholesterol in Adults. Summary of the second report of the National Cholesterol Edury of the second (NCEP). JAMA 1993;269:3015-23.

16 Knopp RH. Drug treatment of lipid disorders. N Engl J Med 1999;341:498-511.

17 Castelli WP, Garrison RJ, Wilson PW, Abbott RD, Kalousdian S, Kannel WB. Incidence of coronary heart disease and lipoprotein cholesterol levels. The Framingham Study. JAMA 1986;256:2835-8.

18 Ramsay LE, Wallis EJ, Hag IU, Yeo WW, Jacobson PR. Sheffield table for primary prevention of cardiovascular disease. Lancet 1998:351:443-4.

19 Walden CE, Retzlaff BM, Buck BL, McCann BS, Knopp $\mathrm{RH}$. Lipoprotein lipid response to the National Cholesterol Education Program Step II Diet by hypercholesterolemic and combined hyperlipidemic women and men. Arterioand combined hyperlipidemic women a

20 Nicholls A, Scott JT. Effect of weight-loss on plasma and urinary levels of uric acid. Lancet 1972;ii:1223-4.
21 Puig JG, Ruilope LM. Uric acid as a cardiovascular risk factor in arterial hypertension. J Hypertens 1999;17:869-72. 22 Facchini F, Chen YD, Hollenbeck CB, Reaven GM. Relationship between insulin-mediated glucose uptake, urinary uric acid clearance, and plasma uric acid concentration. JAMA 1991;266:3008-11.

23 Takahashi S, Yamamoti T, Moriwali Y, Tsutsumi Z, Higashirio K. Impaired lipoprotein metabolism in patients with primary gout-influence of alcohol intake and body weight. Br J Rheumatol 1994;33:731-4.

24 Tinahones JF, Perez-Lindon G, C-Soriguer FJ, Pareja A, Sanchez-Guijo P, Collantes E. Dietary alterations in plasma very low density lipoprotein levels modify renal excretion of urates in hyperuricemic-hypertriglyceridemic patients. J Clin Endocrinol Metab 1997;82:1188-91.

25 Dessein PH, Stanwix AE, Shipton EA, Joffe BI, van der Merwe CA. Dyslipidemia and insulin resistance in gout: sufficiently common to be considered in the evaluation and management of every patient. III. African League Against management of every patient. III. African League Against Rheumatism (AFLAR) Conference. Cape Town, Sor
Africa, September 1999. Cape Town: Ukenza, 1999.

26 Matthews DR, Hosker JP, Rudenski AS, Naylor BA, Treacher DF, Turner RR. Homeostasis model assessment: insulin resistance and beta-cell function from fasting plasma glucose and insulin concentrations in men. Diabetologia 1985;28:412-19.

27 Assmann $G$, Schulte $H$, Funke $H$, von Eckardstein A. The emergence of triglycerides as a significant independent risk ractor in coronary artery disease. Eur Heart J 1998;suppl M:M8-14.

28 Frick MM, Elo O, Haapa K, Meinonen OP, Heinsalmi P, Helo P, et al. Helsinki Heart Study primary-prevention trial with gemfibrozil in middle-aged men with dyslipidemia. Safety of treatment, changes in risk factors, and incidence of coronary heart disease. N Engl J Med 1987;317:123745.

29 Cohen MG, Emmerson BT Crystal-related arthropathies. In: Klippel JH, Dieppe PA, eds. Rheumatology. Vol 8. 2nd ed. London: Mosby, 1998:section 8, 14:1-14. 